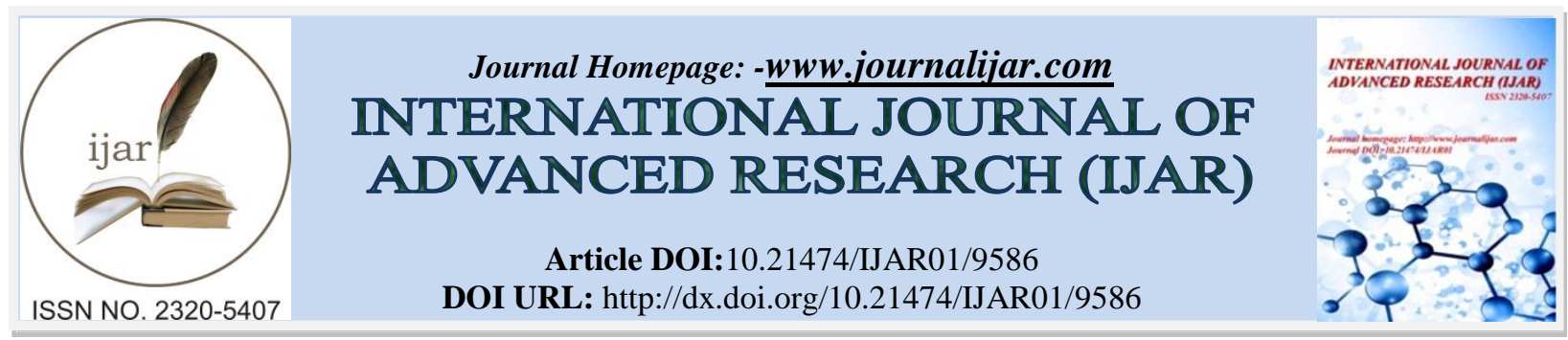

RESEARCH ARTICLE

\title{
SIGNAL TRANSMISSION PERFORMANCE AT 1550NM OVER DIFFERENT FIBER LINKS.
}

\author{
G. K. Yegon ${ }^{1,3}$, D. W. Waswa ${ }^{1}$ G.M. Isoe ${ }^{1,2}$, S. K Rotich $^{3}$, T.B. Gibbon ${ }^{2}$, R.R.G. Gamatham ${ }^{2}$ And A.W.R. \\ Leitch ${ }^{2}$. \\ 1. University of Eldoret, P. O. Box 1125-30100, Eldoret, Kenya. \\ 2. Centre for Broadband Communication, Physics Dept., Nelson Mandela University, South Africa. \\ 3. Moi University, physics department, P. O. Box 3900-30100, Eldoret, Kenya.
}

\section{Manuscript Info}

\section{Manuscript History}

Received: 18 June 2019

Final Accepted: 20 July 2019

Published: August 2019

Keywords

VCSEL, BER, CD.

\begin{abstract}
We experimentally characterized and optimized the performance of a vertical cavity surface-emitting laser (VCSEL) in a communication link. VCSEL at $1550 \mathrm{~nm}$ transmission windows was chosen for data rate of $10 \mathrm{G}$ bps for metro networks due to its wavelength tune ability, cost and energy efficiency. The optical link was evaluated through Bit Error Rate (BER) measurements for $17 \mathrm{~km}$ over G.652 traditional fiber and compared to $24.69 \mathrm{~km}$ of Single Mode Fiber-Reduced Slope (SMF-RS) at $5.5 \mathrm{~mA}$ bias current. The corresponding eye diagrams at telecommunication BER threshold of $10^{-9}$ were captured on the scope. The BER for the two measurements were then compared. Transmission over $24.69 \mathrm{~km}$ on G.655 SMF-RS was achieved with a penalty of $1.39 \mathrm{~dB}$ with respect to B2B. The G.652 fiber at $1550 \mathrm{~nm}$ yields non optimal performance due to its high dispersion coefficient of $17 \mathrm{ps} /(\mathrm{nm} . \mathrm{km})$ and this therefore provided a better platform to compare performance of the two fibers. The maximum transmission distance of $74.91 \mathrm{~km}$ was achieved over G.655 fiber with a penalty of $0.717 \mathrm{~dB}$ with respect to Back to Back (B2B). At transmission distance of $24.69 \mathrm{~km}$ a power penalty of $0.131 \mathrm{~dB}$ was incurred while at $49.42 \mathrm{~km}$ a power penalty of $0.391 \mathrm{~dB}$ was incurred. This therefore proved that shorter distances yielded better results which were observed with high receiver sensitivity. Therefore, with the increase in distance high power is needed to compensate for dispersion effects. This study is vital in enhancing awareness on the effects of $\mathrm{CD}$ on the already laid fibers and hence looking for methods to mitigate the effects for the nextgeneration optical fiber networks.
\end{abstract}

Copy Right, IJAR, 2019,. All rights reserved.

\section{Introduction:-}

A vertical-cavity surface-emitting laser (VCSEL) is a semiconductor-based laser diode that emits a highly efficient optical beam vertically from its top surface. The cavity is formed by two highly reflective Distributed Bragg's Reflector (DBRs) ( 99.5\%), with a forward biased pn-junction sandwiched between them providing optical gain (Haglund, 2015).They have several attractive properties such as large modulation bandwidth, wavelength tune ability, easy packaging and low power consumption(Hofmann et al., 2011).They have therefore become attractive

Corresponding Author:-G. K. Yegon.

Address:-University of Eldoret, P. O. Box 1125-30100, Eldoret, Kenya. 
light sources for high speed optical communication links using intensity modulation/direct detection technique (Chatterjee et al., 2006). VCSELs have gained reputation as a superior technology for short reach applications such as fiber channel, Ethernet and intra-systems links. Optical links that use long-wavelength VCSELs and on-off keying (OOK) modulation are limited in transmission reach by chromatic dispersion of optical fibers due to frequency chirping caused by direct modulation of the VCSEL and large occupied bandwidth of signals (Zhang et al., 2008).

\section{Theory:- \\ VCSEL characteristics:-}

VCSEL used in this experimental work is a $10 \mathrm{G}$ bps $1550 \mathrm{~nm}$. It has low threshold current between 1-2 m A figure 2(a). The VCSEL operate in $\mathrm{m} A$ range showing the energy efficiency of the device. Ease of direct modulation in VCSEL is cost effective as opposed to using external modulation. VCSEL can be tuned to different channels using the bias current. Therefore, if VCSEL with similar characteristics are to be used, then each wavelength within the tune ability range could be dedicated to each device. The wavelength stability and tune ability allows for wavelength multiplexing which is used to increase the data rate over a single fiber in the array. Transmission at $1550 \mathrm{~nm}$ window is currently favoured as it makes use of the minimum attenuation and its application in wavelength division multiplexing (WDM). Generally optical sources emitting at $1550 \mathrm{~nm}$ are suited for use on a G.655 fiber but it can also be used on a G.652 fiber with penalty.

Frequency chirping and dispersion are two major defects experienced by VCSELs during normal operation. Chirping is the instantaneous change of wavelength with varying optical powers. This results in time dependent instantaneous frequency changes. Dispersion dictates how the optical spectrum of a laser broadens during fiber transmission. A number of techniques for reducing and limiting chirping have been reported. These include injection locking and filter off setting to narrow the spectrum. The relationship between chirping and optical power is expressed as (Koch \& Linke, 1986)

$$
\Delta v(t)=-\frac{\alpha}{4 \pi}\left(\frac{d}{d t} \ln P(t)+k P(t)\right)
$$

Where the first term represents the transient chirp that relates to the time derivative of power $(\mathrm{P})$, the second term is the adiabatic chirp due to instantaneous optical power, $\alpha$ is the line enhancement factor and $\mathrm{k}$ is related to the geometry of the device and its non-linear gain.

The material composition of VCSEL used as transmitters in this study are InAlGaAs, InGaAs and InAlAs which offers the VCSEL high reliability, reflectivity, low loss current confinement, reduced thermal impedance and low series resistance for long wavelength emissions. The emission wavelength of VCSEL can be adjusted by varying the drive in bias currents as shown in figure 2(b) below.

\section{Chromatic dispersion:-}

This is the broadening of light pulses over fiber. It is a very critical factor limiting the quality of signal transmission over optical links. Single-mode fibers used in high-speed optical networks are subject to Chromatic Dispersion (CD) that causes pulse broadening depending on wavelength. Excessive spreading will cause bits to "overflow" their intended time slots and overlap adjacent bits (Štěpánek, 2012). The receiver may then have difficulty discerning and properly interpreting adjacent bits, increasing the Bit Error Rate. The Chromatic Dispersion of a fiber is expressed in p s/(nm.km), representing the differential delay, or time spreading (in p s). It depends on the fiber type, and it limits the bit rate or the transmission distance for a good quality of service. Chromatic dispersion coefficient determines the size of the CD and is described by the equation (2) (Štěpánek, 2012)

$$
D(\lambda)=\frac{d t_{g}}{d(\lambda)},\left[\frac{p s}{n m \times k m}\right]
$$

Chromatic dispersion coefficient $D(\lambda)$ expresses group delay $t_{g}$ per km of the signal change per wavelength. The pulse width increases with an increase in coefficient of chromatic dispersion $D(\lambda)$, spectral width of the light source and the length of the fiber. The light components with longer wavelengths are delayed longer in the fiber compared with those of the shorter wavelengths.

\section{Materials and Methods:-}

Experimental setup for high speed VCSEL transmission over different fibers:-

The experimental setup was as shown figure 1 below. The VCSEL biasing was performed by varying the current using the laser diode controller (LDC) and directly modulating the VCSEL with the 10 G bps NRZ PRBS (2 $\left.{ }^{7}-1\right)$ 
Pattern Generator (PPG) via a Bias-Tee (BT). The VCSEL was biased above the threshold current at $5.5 \mathrm{~m}$ A. The modulated signal from the VCSEL was then transmitted over the fiber under test (FUT). In this experiment, the FUT comprised of $17 \mathrm{~km} \mathrm{G.652} \mathrm{fiber} \mathrm{and} 24.69 \mathrm{~km}$ of G.655 fiber. A variable optical attenuator (VOA) was used to vary the optical signal power entering the photodiode to mimic the typical losses in a fiber link. The receiver consisted of Positive Intrinsic Negative (PIN) photodiode and an Electrical Amplifier (EA). This EA was used to amplify the electrical signal to meet the operational requirements of a (Bit Error Rate Tester) BERT. The quality of the transmitted signal was then evaluated by the eye diagram and BER measurements using an oscilloscope and BERT respectively. The BERT determines the number of errors received and compares the errors to the total number of transmitted bits. A clock signal from the PPG was used to synchronize the $10 \mathrm{G}$ bps transmitted and received data signals. Then finally the quality of the optical signal was characterized by observing the electrical data eye diagrams, BER measurements and penalties. The optical communication system tolerance to the effects of dispersion were measured for the two fibers. The G.652 fiber used has low attenuation but has very high CD parameter of $17 \mathrm{p}$ s/nm.km at $1550 \mathrm{~nm}$ compared to $0.35 \mathrm{p} \mathrm{s} / \mathrm{nm} . \mathrm{km}$ at $1310 \mathrm{~nm}$.

\section{Intensity Modulation at $1550 \mathbf{~ n m}$ window at different fiber lengths:-}

In this setup the laser operating at $1550 \mathrm{~nm}$ was used. A Non Return Zero (NRZ) pulse generator which operates as a laser driver is modulated by a pseudorandom bit sequence (PRBS) generator. The laser was then connected to the Mach-Zender Modulator (MZM) via Polarization Coupler (PC) which was then connected to the SMF. The modulated signal was then transmitted through the fiber. The different fiber-links used were made up of SMF G.652 and G.655 True-Wave-Reduced slope (TW-RS) fiber()(7)(). The G.652 fiber had a 17 p s/(nm.km) while the G.655 fibers had a coefficient of $4.5 \mathrm{p} \mathrm{s} /(\mathrm{nm}$. $\mathrm{km})$ dispersion coefficients at the $1550 \mathrm{~nm}$ transmission window. Moreover, the PMD coefficient of G.652 and TW-RS G.655 fibers are $\leq 0.2 \mathrm{ps} / \mathrm{km}^{2}$ and $\leq 0.1 \mathrm{ps} / \mathrm{km}^{2}$ respectively. G.655 is optimized for $1550 \mathrm{~nm}$ transmission window where it experiences minimum chromatic dispersion. The fiber output was then connected to a variable optical attenuator (VOA) which varies the optical power to emulate typical power loss in a fiber link.

The output was then fed to an optical receiver Positive Intrinsic Negative (PIN) Photodiode with the error-free sensitivity of $-19 \pm 1 \mathrm{~dB}$ for $10 \mathrm{G}$ bps at $10^{-9}$ BER threshold. The optical power was then varied so as to establish bit errors at different power levels. The electrical output from the PIN was then connected to an EA to boost the signal so as to satisfy BERT operation voltage. A data recovery module is connected at the end of the link, in order to observe and measure the quality of the link through its eye diagram and bit-error rate (BER) characteristics. The binary signal input of the data recovery module was connected to the PRBS (a pseudo random binary sequence was used as a model data to test a high-speed serial interface device for emulating a transmission mode) output and the electrical signal input was connected to the sequence generator. This was to enable the data recovery module to compare the transmitted and received signal for BER analysis. The optical power meter was connected to the attenuator output to measure the received optical power (known as sensitivity at BER $=10^{-9}$ ).

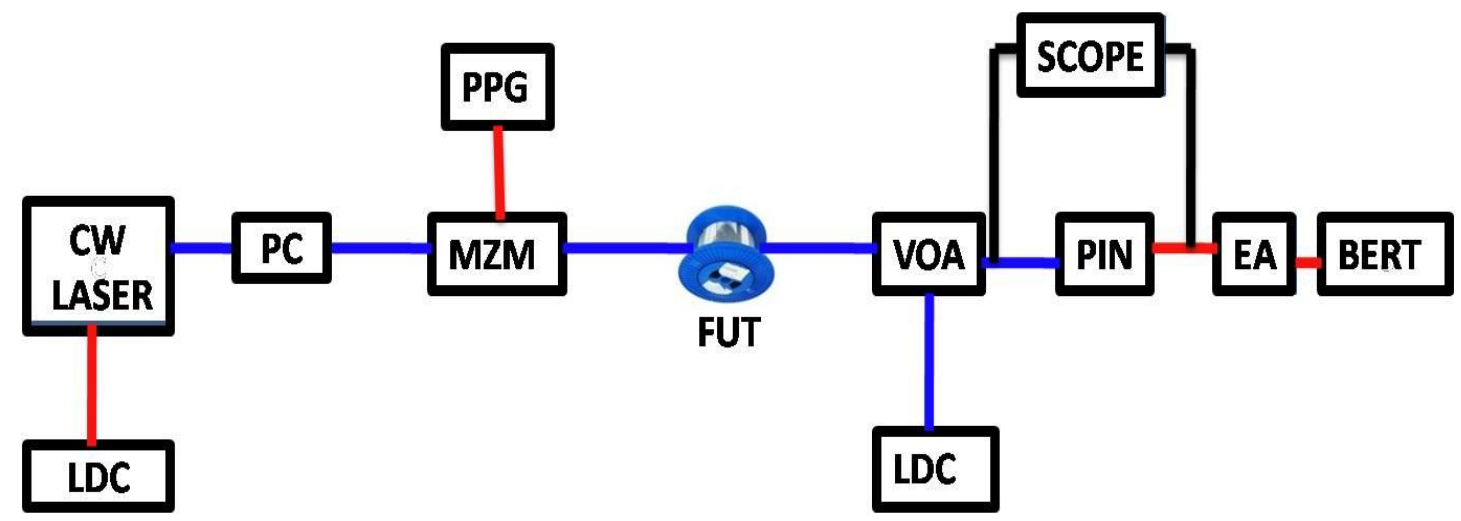

Figure 1:-External Intensity modulation at $1550 \mathrm{~nm}$ using the $\mathrm{MZ}$

\section{Results and discussions:-}

VCSEL transmission over G.652 and G.655 optical fibers:-

To evaluate the system performance, BER measurements as a function of the received power were performed for B2B, $17 \mathrm{~km}$ over G.652 fiber and transmission over 24.69 km SMF-RS as shown in the figure 2 below. 

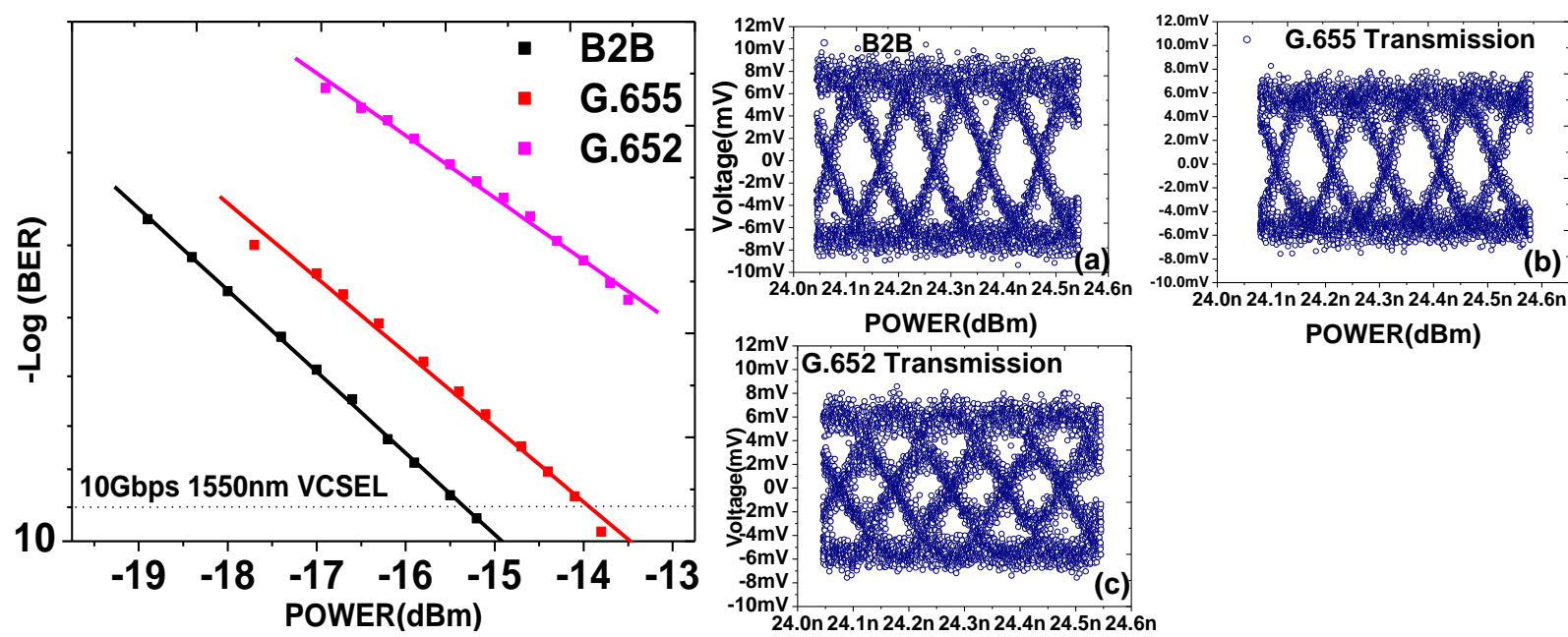

Figure 2:-1550 nm VCSEL transmission on different fibers (a) B2B (b) 24.69km G.655 SMF-RS fiber (c) $17 \mathrm{~km}$

G.652 fiber

Transmission over $24.69 \mathrm{~km}$ on G.655 SMF-RS was achieved with a penalty of $1.39 \mathrm{~dB}$ at BER of $10^{-9}$ with respect to B2B.An error free transmission was achieved for $24.69 \mathrm{~km} \mathrm{SMF-RS} \mathrm{before} \mathrm{getting} \mathrm{an} \mathrm{error} \mathrm{transmission} \mathrm{for}$ G.652 fiber. The error transmission is graphically indicated by the failure of the BER graph to cross the minimum threshold BER of $10^{-9}$ as shown above. The error floor is independent of the received optical power; hence no matter how much optical is introduced into the system it will never cross the minimum threshold BER value. Despite increase in power, the transmission distance is limited by high linear penalties. In this setup the error floor was mainly attributed to the high CD parameter of $17 \mathrm{ps} / \mathrm{nm} . \mathrm{km}$ for the G.652 at $1550 \mathrm{~nm}$ wavelength which resulted in increased number of error-bits received. It's therefore evident that $1550 \mathrm{~nm}$ signal transmission are best suited for long distance G.655 fiber since dispersion effects are lower at $1550 \mathrm{~nm}$ transmission window. The eye diagram for B2B, transmission over $24.9 \mathrm{~km}$ G.655 SMF-RS and $17 \mathrm{~km}$ G.652 SMF in the above diagrams clearly show the effect of dispersion on the modulated signal. Increasing the distance to $24.69 \mathrm{~km}$ induces a penalty of $1.39 \mathrm{~dB}$ in receiver sensitivity. A wide and open eye represented an error free transmission. When the eye was open the receiver was able to distinguish between the 1's and 0's and therefore few errors were detected. The eye diagram for G.652 is smaller than for G.655fiber though it has a shorter length. This was due to high dispersion coefficient of G.652 on $1550 \mathrm{~nm}$ window at $17 \mathrm{p} \mathrm{s} / \mathrm{nm}$. km. It was also noted in figure (c) above that the eye diagram does not have a good separation of signal levels. The big number of values between the lowest and the highest levels was the source of blurred diagram and was due to high chromatic dispersion on G.652 at $1550 \mathrm{~nm}$ window. It's therefore clear that the G.655 fiber is optimized for $1550 \mathrm{~nm}$ transmission window.

Transmission at 1550nm window at different lengths:-
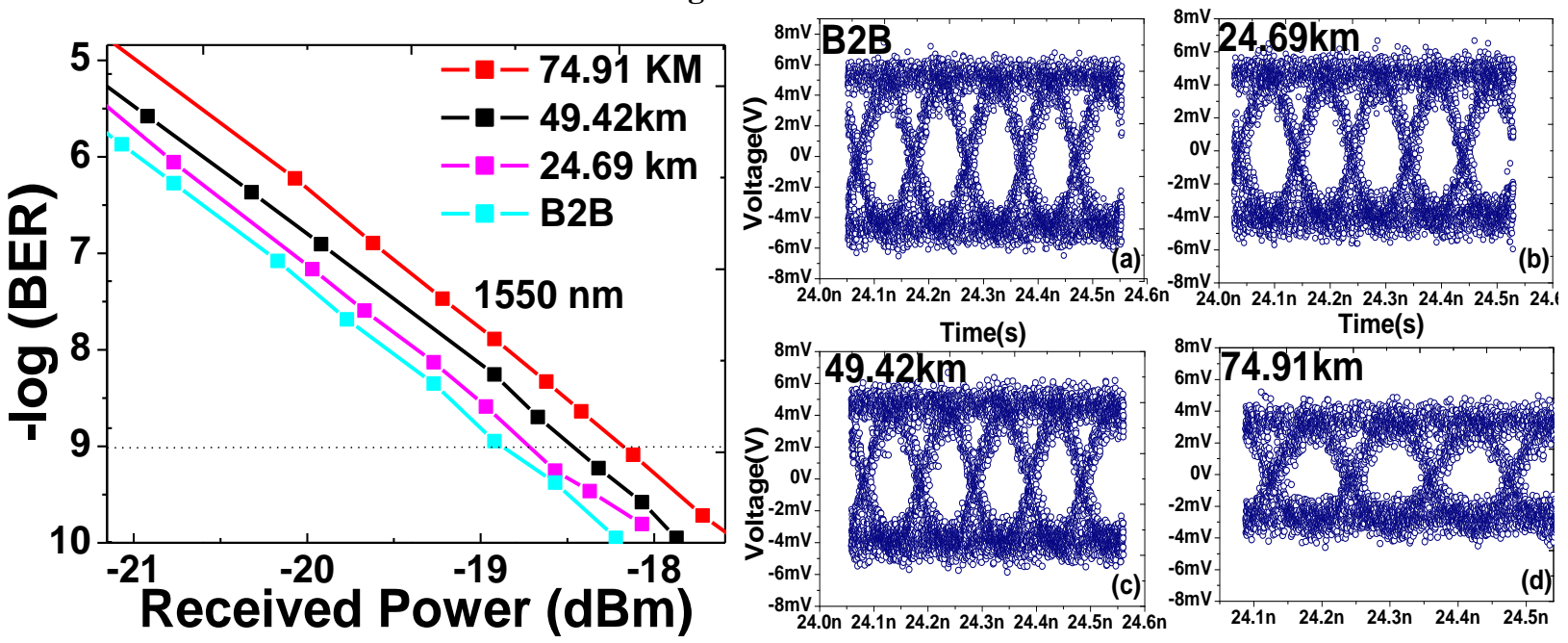

Figure 3:-BER graphs and their eye diagrams at different lengths for 10Gbps 
Figure 3 shows the BER curves at different lengths for different fiber combinations. For B2B the receiver sensitivity was observed at $-18.858 \mathrm{~dB}$. When the signal was transmitted through a distance of $24.69 \mathrm{~km}$ over a SRS fiber the receiver sensitivity was observed to be $-18.727 \mathrm{~dB}$ which gives a power penalty of $0.131 \mathrm{~dB}$ with respect to the B2B. When the transmission distance was increased to $49.42 \mathrm{~km}$ of SRS and RS fiber the receiver sensitivity of $-18.467 \mathrm{~dB}$ was attained with a power penalty of $0.391 \mathrm{~dB}$ with respect to B2B while at $74.91 \mathrm{~km}$, the receiver sensitivity was observed to be $-18.141 \mathrm{~dB}$ giving a power penalty of $0.717 \mathrm{~dB}$ with respect to B2B. This increase in power penalty as the distance increases signifies the power loss due to the effects of dispersion along the fiber link. The eye diagrams for B2B, transmission over $24.69 \mathrm{~km} \mathrm{SRS}, 49.42 \mathrm{~km} \mathrm{(24.69} \mathrm{km} \mathrm{SRS} \mathrm{+24.73} \mathrm{km} \mathrm{RS)} \mathrm{and} 74.91 \mathrm{~km}(24.69 \mathrm{~km} \mathrm{SRS}$ $+24.73 \mathrm{~km} \mathrm{RS}+25.49 \mathrm{~km}$ SRS) SMF are clearly shown in the above diagrams. Because of the dispersion along the link, the eye tends to close with increasing transmission distance, degrading the signal quality. This can be attributed to the fact that increase in distance resulted in pulse broadening and hence increase in bit errors due to the overlapping of bits. However, even at $74.91 \mathrm{~km}$ a sufficient open eye was still obtained with an error free performance at BER of $10^{-9}$. Increasing the distance to $74.91 \mathrm{~km}$ induces a penalty of $0.717 \mathrm{~dB}$ in receiver sensitivity

\section{Conclusions:-}

We have successfully demonstrated chromatic dispersion using VCSELs over 17 km G.652 and 24.69 km G.655 fiber link at $1550 \mathrm{~nm}$ transmission window. The optical communication system tolerance to the effects of dispersion was measured for two different types of fibers at different. As a result, chromatic dispersion based on vertical-cavity surface emitting lasers (VCSELs) has been successfully demonstrated. Since G.655 fibers are optimized for $1550 \mathrm{~nm}$ transmission window, transmissions over G.652fiber yielded a non-optimal performance due to the high effects of $\mathrm{CD}$ and therefore gave a better platform to compare the performance of the two fibers. An experimental analysis of the performance degradation due to Fiber length was also demonstrated, revealing how increasing the length of the fiber affects the BER performance. It was therefore noted that there was better receiver sensitivity at shorter lengths. This was attributed to the fact that at long distances there is cumulative dispersion effects and hence high power is required to compensate for the same. Therefore, for any communication channel, transmission of undistorted signal to the end user remains the primary role. The Quality of Signal and Quality of Transmission should always be maintained when the signal is transmitted for longer distances. This requires that the transmitters, fibers and receivers are all optimized for realization of better end-to-end transmission.

\section{Acknowledgement:-}

We are grateful for research funding and support from: Nelson Mandela University, African Laser centre (ALC) and University of Eldoret, Kenya.

\section{References:-}

1. Chatterjee, S., Wohlleben, W., Lange, C., Stolz, W., Motzkus, M., \& Ruhle, W. (2006). Optimizing the performance of a vertical-cavity surface-emitting laser after optical excitation. Paper presented at the Lasers and Electro-Optics, 2006 and 2006 Quantum Electronics and Laser Science Conference. CLEO/QELS 2006. Conference on.

2. Haglund, E. (2015). VCSELs for High-Speed, Long-Reach, and Wavelength-Multiplexed Optical Interconnects: Chalmers University of Technology.

3. Hofmann, W., Müller, M., Wolf, P., Mutig, A., Gründl, T., Böhm, G., Amann, M.-C. (2011). 40 Gbit/s modulation of $1550 \mathrm{~nm}$ VCSEL. Electronics Letters, 47(4), 270-271.

4. Koch, T., \& Linke, R. (1986). Effect of nonlinear gain reduction on semiconductor laser wavelength chirping. Applied Physics Letters, 48(10), 613-615.

5. Štěpánek, L. (2012). Chromatic dispersion in optical communications. International Journal of Modern Communication Technologies \& Research, 7.

6. Zhang, B., Zhao, X., Christen, L., Parekh, D., Hofmann, W., Wu, M. C., Willner, A. E. (2008). Adjustable Chirp Injection-Locked 1.55-n m VCSELs for Enhanced Chromatic Dispersion Compensation at 10-Gbit/s. Paper presented at the Optical Fiber Communication Conference. 